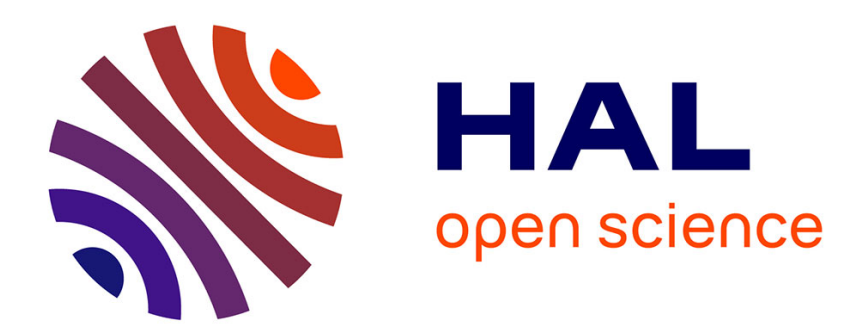

\title{
Les recherches à propos de l'enseignant: des images entre prescription et description
}

Philippe Dessus

\section{To cite this version:}

Philippe Dessus. Les recherches à propos de l'enseignant: des images entre prescription et description. Recherche et formation, 1995, 20, pp.33-43. hal-01297174

\section{HAL Id: hal-01297174 \\ https://hal.science/hal-01297174}

Submitted on 3 Apr 2016

HAL is a multi-disciplinary open access archive for the deposit and dissemination of scientific research documents, whether they are published or not. The documents may come from teaching and research institutions in France or abroad, or from public or private research centers.
L'archive ouverte pluridisciplinaire HAL, est destinée au dépôt et à la diffusion de documents scientifiques de niveau recherche, publiés ou non, émanant des établissements d'enseignement et de recherche français ou étrangers, des laboratoires publics ou privés. 


\title{
LES RECHERCHES À PROPOS DE \\ L'ENSEIGNANT : DES IMAGES ENTRE PRESCRIPTION ET DESCRIPTION
}

\author{
Philippe DESSUS $*$
}

\begin{abstract}
La littérature de Sciences de l'éducation à propos de l'enseignant regorge d'images parfois contradictoires, souvent conflictuelles, sur ce qu'il est ou devrait être. Ces images peuvent être classées en deux grandes approches : descriptive/prescriptive et décrivent un enseignant rationnel ou arationnel.

Sont décrits, puis commentés, le courant de la professionnalisation et son versant descriptif : l'enseignant réfléchi ; le courant tylerien et son pendant : l'enseignant planificateur et enfin un courant bivalent, celui de l'enseignant décideur. Pour terminer, nous ouvrons une discussion sur les images de l'enseignant qui seront vraisemblablement débattues dans les années à venir et qui devront sans doute concilier les approches prescriptives et descriptives.
\end{abstract}

Mots-clés : Pensée des enseignants, recherche descriptive et prescriptive, rationalité, planification, décision.

The literature of teachers' thinking has plenty of metaphors, occasionally inconsistent, often conflicting, about the teacher is or would be. These metaphors fall into two approaches: descriptive vs prescriptive, and about teacher's rationality vs arationality.

We describe and comment, at once teacher as a professional vs a reflective practitioner, then as a tylerian-based approach of planning vs a problem-solving approach; finally, teacher as a decision-maker. We conclude by discussing the future metaphors, which would be able to reconcile the prescriptive and descriptive approaches.

Key words : Teachers' thinking, descriptive and prescriptive research, rationality, planning, decision-making.

L'enseignement est une profession reposant sur des «paradigmes conflictuels » (1). Cela pourrait être un signe de scientificité de la recherche en éducation si, comme l'indique Shulman (2), un seul paradigme à la fois était partagé par tous les

* Lab. Sciences de l'éducation, Université de Grenoble II. Paru in : Dessus, P. (1995). Les recherches à propos de l'enseignant : des images entre prescription et description. Recherche et formation, 20, 33-43. 96.

(1) BOURDONCLE, R. (1994). Savoir professionnel et formation des enseignants. Spirale, 13, $77-$

(2) SHULMAN, L. S. (1986). Paradigms and research programs in the study of teaching : a contemporary perspective. In M. C. WITTROCK (Ed.), Handbook of research on teaching. New York : McMillan, 3-36. 
chercheurs, ce qui, à notre avis, n'est guère le cas. Des paradigmes concurrents s'affrontent, sont repris, abandonnés selon les courants de recherche, et il est souvent difficile de s'y retrouver. Cet article (3) va être l'occasion de reprendre et commenter les différentes images de l'enseignant que les chercheurs en éducation ont pu adopter.

Commençons par décrire ce qui semble recueillir le plus large consensus. Les chercheurs s'accordent à poser que l'enseignant travaille face à un environnement dynamique (il peut évoluer sans son intervention), met en œuvre diverses activités (recherche, sélection, représentation et combinaison d'informations, décisions, mise en œuvre de routines, jugements, évaluations, etc.) et traite des informations de nature différente (représentations, anticipations, souvenirs, croyances, etc.) pour agir sur cet environnement. Ce triptyque environnement-activités-informations permet de décrire assez fidèlement le processus cognitif d'un enseignant. Ce dernier se trouverait devant des situations qualifiées de problématiques, mais les buts poursuivis et les moyens utilisés pour tenter de résoudre ces problèmes diffèrent largement selon les approches.

Qu'ils soient de nature ergonomique, cognitiviste, sociologique, éducative ou didactique, la majorité des travaux produits a eu pour but ou pour conséquence de rationaliser le travail de l'enseignant (4). Si l'on considère ce qui divise les chercheurs, on peut distinguer deux grands axes : l'approche prescriptive/descriptive, qui semble également répartie dans la littérature ; le présupposé de l'enseignant rationnel/arationnel qui, lui, peut fluctuer selon les facteurs des expérimentations.

Il va de soi que ces images ne sont pas créées ex nihilo, mais correspondent à des finalités diverses :

- le premier axe concerne le versant descriptif de la recherche, afin d'élaborer des modèles reproduisant aussi fidèlement que possible l'activité de l'enseignant ;

- le second axe définit plutôt ce que doit être le processus d'enseignement, ce côté prescriptif étant notamment utile dans le domaine politique ou de la formation des enseignants.

Nous allons décrire ces deux approches, puis nous nous pencherons sur les principales images que l'on peut rencontrer dans la littérature à propos des enseignants. Nous terminerons en signalant ce que les recherches futures pourront apporter à l'étude des processus de pensée de l'enseignant.

(3) Merci à Jean-Jacques Maurice qui en a fait une lecture vigilante et avisée ; nous restons bien évidemment responsable des erreurs qui auront pu subsister.

(4) Voir notamment PERRENOUD, P. (1988). La pédagogie de maîtrise, une utopie rationaliste ? In M. HUBERMAN (Ed.), Assurer la réussite des apprentissages scolaires ? Neuchâtel : Delachaux et Niestlé, 198-233 ; et TOCHON, F. V. (1993). L'enseignant expert. Paris : Nathan. 


\section{L'APPROCHE PRESCRIPTIVE VS DESCRIPTIVE}

Bru, en distinguant les modèles de l'enseignement et les modèles pour l'enseignement (5), nous permet d'opérer une distinction claire entre deux sortes d'images. Celles qui appartiennent au domaine prescriptif (pour l'enseignement) ne se soucient pas de validité, mais essaient de définir ce que pourrait être un bon enseignant, une bonne préparation de classe, une bonne école. Prime ici l'efficacité, dans la formation des enseignants, par exemple. Celles qui appartiennent au domaine descriptif, elles, essaient d'être plus près de la réalité, gagnant en explicativité ce qu'elles perdent en désirabilité sociale.

Ce critère de classement n'est toutefois pas assez fin pour rendre compte de toutes les images que nous avons inventoriées. Les préoccupations des chercheurs ont également eu pour objet de restreindre la complexité des conduites de l'enseignant et, par la même occasion, de tenter d'y repérer une certaine régularité.

\section{L'ENSEIGNANT RATIONNEL VS ARATIONNEL}

Le deuxième critère est donc d'observer une (a-)rationalité chez l'enseignant. Il s'agit de tester si la pensée de l'enseignant est prédictible, reproductible, afin de la modéliser. Tâche ardue, voire irréalisable, car, pour reprendre Martel (6), si l'on parle tant de rationalité à propos du décideur, « c'est justement parce qu'on se rend compte qu'il ne se comporte pas de façon toujours rationnelle $»$ (p. 45).

Un enseignant rationnel pense que, pour chaque situation d'enseignement, il en est au moins une qui conviendra à ses élèves. Il suppose qu'il a les moyens de la mettre en œuvre, étant face à une situation déterministe dont l'évolution est presque totalement prévisible en fonction de son activité. Face à une alternative, il décide de la meilleure action possible et contrôle à chaque instant l'évolution de la situation. Comme un joueur d'échecs, il est capable de prévoir de nombreux coups à l'avance ainsi que les réponses respectives à y apporter. Ce modèle idéal, provenant des théories mathématiques de la décision (7) est bien entendu battu en brèche par bon nombre de travaux, qui concluent, tout au plus, à une éventuelle rationalité limitée de l'enseignant (8).

(5) BRU, M. (1994). Quelles orientations pour les recherches sur la pratique de l'enseignement? L'année de la recherche en Sciences de l'éducation, 1, 165-174.

(6) MARTEL, J.-M. (1983). Débat à propos du texte de M. Crozier « La rationalité du décideur du point de vue du sociologue ». In B. ROY (Ed.), La décision, ses disciplines, ses acteurs. Lyon : P.U.L., 45-46.

(7) Pour une introduction, voir notamment CHARRETON, R., BOURDAIRE, J.-M. (1985). La décision économique. Paris : P.U.F. ; COOMBS, C. H., DAWES, R. M., TVERSKY, A. (1975). Psychologie mathématique. Paris : P.U.F ; et KAST, R. (1993). La théorie de la décision. Paris : La Découverte.

(8) LEE, O., PORTER, A. C. (1993). A teacher's bounded rationality in middle school science. Teacher and Teaching Education, 9-4, 397-409. 
À l'opposé se trouverait un enseignant arationnel qui, sans être irrationnel, prendrait des décisions compilées extrêmement dépendantes du contexte, de sorte qu'elles seraient difficiles à modéliser. Il semble que cette caractéristique serait le propre des enseignants expérimentés, voire experts.

Après avoir rapidement brossé les différentes caractéristiques de ces approches, nous pouvons essayer de décrire quelques images apparaissant dans la littérature : l'enseignant professionnel et son versant descriptif : l'enseignant réfléchi ; l'image prescriptive de l'enseignant tylerien et son pendant, l'enseignant planificateur et enfin un courant bivalent, en partie descriptif et prescriptif, celui de l'enseignant décideur.

\section{L'ENSEIGNANT PROFESSIONNEL}

Le terme « enseignant professionnel » sonne comme un pléonasme : en quoi pourrait-il être considéré comme un amateur ? «Profession » n'est pas ici à opposer à amateurisme, mais à «métier» (9). Ainsi, «profession» renvoie à un travail plutôt prestigieux, alors que métier n'est souvent associé qu'à un travail mécanique, voire manuel. Une profession est, d'après le Bureau international du travail, cité par Legendre (10) une

« activité professionnelle de caractère intellectuel, dont l'exercice exige des connaissances et des compétences de haut niveau, généralement acquises dans le cadre d'études supérieures, universitaires ou autres, dans des domaines tels que le droit, la médecine, la physique, les mathématiques, les professions d'ingénieur, etc. » (p. 1028).

En devenant universitaire, la formation des enseignants tente de professionnaliser ce qui, auparavant, était un métier. Shavelson (11) résume parfaitement notre propos :

«Tout d'abord, les enseignants sont des professionnels rationnels qui, comme d'autres professionnels tels que les physiciens, émettent des jugements et prennent des décisions dans un environnement complexe et indéterminé. [...] les enseignants se comportent rationnellement en ce qui concerne les modèles simplifiés de la réalité qu'ils construisent. [...] le comportement des enseignants est guidé par leurs pensées, jugements et décisions. » (p. 23).

Ainsi, les bénéfices que l'on essaie de retirer de cette vision professionnelle de l'enseignant sont loin d'être seulement sociaux ou politiques. Les chercheurs pensent

(9) Voir TROUSSON, A. (1992). De l'artisan à l'expert, la formation des enseignants en question. Paris : C.N.D.P./Hachette.

(10) LEGENDRE, R. (1993). Dictionnaire actuel de l'éducation. Montréal/Paris : Guérin/Eska.

(11) SHAVELSON, R. J. (1983). Review of research on teachers' pedagogical judgements, plans and decisions. Elementary school Journal, 83-4, 392-413. ; cité par SHULMAN, L. S. (op. cit.). 
pouvoir appliquer à ces professionnels les techniques d'analyse couramment utilisées pour des professions «techniques ». Issue de l'approche sociologique, cette démarche postule que l'enseignant a des comptes à rendre à son entourage, parents, élèves, collègues, supérieurs ; on reconnaît ou non ses capacités à enseigner. Il développe un savoir-faire conjointement issu de son enseignement universitaire de haut niveau et de son expérience, voire de son expertise.

Labaree (12) dénonce quelques effets paradoxaux de ce mouvement. Tout d'abord, il augmente l'influence de l'université sur l'enseignement primaire et secondaire, en donnant beaucoup plus de poids à l'autorité des formateurs des enseignants et il augmente la rationalisation de l'enseignement en imposant le modèle de la rechercheaction, ces deux facteurs ayant pour effet de réduire, selon Labaree, le poids de l'enseignant et du citoyen au sein du système éducatif.

L'image de l'enseignant professionnel est une heuristique pour mieux penser la formation des enseignants ou bien leur nouvelle place dans la société, en aucun cas cela n'est un modèle de leur activité. La métaphore de l'enseignant réfléchi semble avoir été conçue pour pallier ce problème.

\section{L'ENSEIGNANT RÉFLÉCHI}

Pendant descriptif de la métaphore précédente, l'image de l'enseignant réfléchi lui fait privilégier son auto-évaluation. Ce dernier puise dans sa propre pratique les éléments qui vont lui permettre de s'analyser et d'aller vers un meilleur enseignement. De nombreux modèles de formation initiale s'appuient sur cette hypothèse (13). Sans pour autant dénier que l'enseignant est face à une situation problématique et développerait des stratégies de résolution de problème, les études sur l'enseignant réfléchi adoptent une vision plutôt ethnographique, où le contexte social prime l'utilisation de schémas d'actions.

L'enseignant réfléchi s'engagerait donc — et c'est en cela qu'il se rapprocherait de l'enseignant planificateur - , lors de situations ou de contextes qui lui semblent problématiques, dans un processus de résolution de problèmes - identification du problème, génération de solutions possibles et tests de ces solutions, évaluation en termes

(12) LABAREE, D. F. (1992). Power, knowledge, and the rationalization of teaching : a genealogy of the movement to professionalize teaching. Harvard Educational Review, 62-2, 123-154.

(13) MORINE-DERSHIMER, G. (1989). Preservice teachers' conceptions of content and pedagogy : measuring growth in reflective, pedagogical decision-making. Journal of Teacher Education, 40-5, 47-52 ; et ZEICHNER, K. M. (1987). Preparing reflective teachers : an overview of instructional strategies which have been employed in preservice teacher education. International Journal of Educational Research, 11-5, 565-575. 
d'apprentissage, mémorisation (14). Ainsi, c'est plus la réflexion-en-action que sur l'action qui sera recherchée et favorisée.

Les critiques, bien évidemment, ne manquent pas. Certains dénoncent la survalorisation de l'image de l'enseignant-chercheur (15). D'autres lui reprochent sa centration sur l'introspection, dont la psychologie ergonomique a pu mesurer les inconvénients. Rien ne prouve en effet que l'enseignant face à une double tâche - action et réflexion — se comporte comme s'il avait seulement à enseigner.

Passons maintenant à la principale approche concurrente au courant de l'enseignant professionnel/réfléchi, plutôt arationnel : celle qui consiste à techniciser la spécification des séquences d'enseignement : l'enseignant tylerien/planificateur, se réclamant d'une approche essentiellement rationnelle.

\section{L'ENSEIGNANT TYLERIEN}

L'image de l'enseignant tylerien est sans doute la vision la plus répandue dans la littérature et il n'est pas dans notre propos de rendre compte exhaustivement des conclusions qu'elle a pu engendrer. Il faut noter, toutefois, que l'enseignant tylerien est une approche essentiellement prescriptive, depuis que Zahorik (16) a montré que l'enseignant ne commençait pas par spécifier les objectifs préalablement à ses leçons. Ce décalage avec la réalité n'a pas empêché quantité de littérature consacrée à cette image de l'enseignant, dépassant largement les autres (17).

L'enseignant tylerien n'aura de cesse de présenter son travail de la façon la plus séquentielle, programmée possible. Il formule très rigoureusement les activités des élèves, les siennes propres, en se centrant sur le comportement. Mais comme il est toujours possible de segmenter une tâche en atomes comportementaux de plus en plus fins,

(14) COPELAND, W. D., BIRMINGHAM, C., DE LA CRUZ, E. (1993). The reflective practitioner in teaching : toward a research agenda. Teacher and Teaching Education, 9-4, 347-359.

(15) VAN DER MAREN, J.-M., BLAIS, J.-G. (1994). Quelle recherche et avec quel rôle dans la formation professionnelle en éducation ? Recherche et formation, 17, 123-140. $143-151$

(16) ZAHORIK, J. A. (1970). The effect of planning on Teaching. Elementary School Journal, 71,

(17) À propos de la seule détermination des objectifs de l'enseignement, voir BIRZÉA, C. (1979). Rendre opérationnels les objectifs pédagogiques. Paris : P.U.F. ; HAMELINE, D. (1979). Les objectifs pédagogiques en formation initiale et en formation continue. Paris : E.S.F. ; MAGER, R. (1974). Comment définir des objectifs pédagogiques. Paris : Bordas ; POCZTAR, J. (1977). La définition des objectifs pédagogiques. Paris : E.S.F. ; VAN DE VELDE, L., VAN DER ELST, P. (1975). Peut-on préciser les objectifs en éducation? Bruxelles : Labor.

Pour une approche plus générale et néanmoins très « technologique » de l'enseignement, se référer à BERTRAND, Y. (1993). Théories contemporaines de l'éducation. Lyon : Chronique sociale ; BRIEN, R. (1993). La planification d'activités de formation, vers l'essentiel. In R. VIAU (Ed.), La planification de l'enseignement. Québec : CRP, 25-37 ; LEBRUN, N., BERTHELOT, S. (1991). Design de systèmes d'enseignement. Ottawa : A.R.C. ; PRÉGENT, R. (1993). La planification de l'enseignement en milieu universitaire. In R. VIAU (Ed.), La planification de l'enseignement. Québec : CRP, 39-62. 
sa planification reste par essence incomplète. Malglaive (18) porte à ce sujet une des critiques les plus pertinentes : la formule invoquée lors de planifications, « l'élève doit être capable de...» a trait à l'activité finale de l'élève et non à sa tâche. Cela n'éclaire donc en rien l'enseignant sur la tâche que l'élève doit mettre en œuvre pour, finalement, développer la capacité invoquée.

Cette approche n'est toutefois pas totalement néfaste et permet aux enseignants, élèves, inspecteurs, parents d'élèves, voire à la société, de comprendre et réguler aisément le processus d'enseignement-apprentissage (19). Son versant descriptif, que nous présentons maintenant, tente de décrire l'activité de la «boîte noire » mentale de l'enseignant.

\section{L'ENSEIGNANT PLANIFICATEUR}

La métaphore de l'enseignant planificateur est issue de l'approche « traitement de l'information » en cours dans la psychologie cognitive. La planification serait une composante importante du processus d'enseignement et cette approche rend compte de la création, utilisation, modification de routines - procédures comportementales acquises — chez les enseignants.

Ces routines ont vraisemblablement pour rôle de maintenir l'enseignant dans un état d'efficacité cognitive pendant son activité et d'accroître sa flexibilité dans un contexte changeant. L'enseignant planificateur raisonne comme s'il avait à résoudre des problèmes. S'il possède une routine appropriée au problème, il l'exécute, sinon, il adopte une stratégie de type « résolution de problème » afin d'y faire face, selon les contraintes qui se présentent à lui.

De nombreuses études (20) montrent que l'hypothèse de l'enseignant planificateur peut s'avérer ; il parcourrait un espace de problèmes, sélectionnant un ou plusieurs schéma(s) d'action en fonction de la tâche qui lui est donnée et particularisant ce(s) schéma(s) selon le contexte. Néanmoins, la critique majeure que l'on peut faire à cette approche porte sur l'accès des chercheurs aux routines : procéduralisées, elles ne sont pas accessibles sous forme déclarative sans biais importants.

(18) MALGLAIVE, G. (1990). Enseigner à des adultes. Paris : P.U.F.

(19) BERGER, G. (1982). Technologie et behaviorisme, une rencontre essentielle et malencontreuse. In M. TARDY (Ed.), Les formes médiatisées de la communication éducative : Actes du colloque du centenaire de l'E.N.S. de Saint-Cloud, 95-103.

(20) LEINHARDT, G., GREENO, T. (1986). The cognitive skill of teaching. Journal of Educational Psychology, 78-2, 75-95 ; LOWYCK, J. (1984). Teacher thinking and teacher routines : a bifurcation? In R. HALKES, J. K. OLSON (Eds), Teacher Thinking. Lisse : Swets et Zeitlinger, 7-18; YINGER, R. (1979). Routines in Teacher Planning. Theory into practice, 18-3, 163-169. 


\section{L'ENSEIGNANT DÉCIDEUR}

L'image de l'enseignant décideur, issue des théories économiques de la décision dans l'incertitude occupe une place à part dans la littérature. Initiée par Shavelson (21), elle s'inspire de la théorie mathématique de la décision, prescriptive ; et essaie de repérer des régularités dans le comportement de l'enseignant, ce qui est un but descriptif. Ici, on s'intéresse plus au contexte de classe qu'à la planification. Il s'agit pour l'enseignant de repérer des indices de l'environnement afin de prendre les décisions appropriées. Deux types d'enseignants décideurs peuvent être modélisés, l'enseignant utilitariste et l'enseignant bayesien (22) :

- l'enseignant utilitariste est sensé effectuer le choix qui maximise son utilité attendue. Il modélise l'activité de l'élève en probabilités d'occurrences de comportements et en utilités (notes subjectives), attribuant une valeur à chaque éventualité ;

- l'enseignant bayesien prélève des informations dans l'interactif, il échantillonne les comportements, les résultats des élèves afin de décider ultérieurement de la meilleure stratégie. La réussite d'un nombre réduit d'élèves serait un bon prédicteur de la réussite de l'ensemble de la classe.

L'enseignant décideur " travaille sans filet », privilégie l'interaction avec les élèves à la préparation de ses leçons. Attentif à tout ce qui se passe en classe, il est capable de prendre de nombreuses décisions. Il vit en direct les réactions de ses élèves, les interprétant comme autant de signaux révélateurs d'échec ou de réussite.

Malgré certains résultats (23) qui auraient peut-être mérité une réplication, ce courant n'est pratiquement plus étudié à ce jour (24). Une des critiques majeures que l'on peut faire à l'encontre de ce modèle est que la falsification de son hypothèse principale est difficile. Il reste souvent possible de montrer que les décisions prises par les sujets sont peu différentes des décisions prédites par le modèle. Il est certain qu'ainsi elles ne répondent pas au critère classique de falsifiabilité, où une hypothèse doit être infirmée plutôt que confirmée.

(21) SHAVELSON, R. J. (1973). What is the basic teaching skill ? Journal of Teacher Education, $24,144-151$.

(22) DESSUS, P., MAURICE, J.-J., NEZ, A., BAILLÉ, J. (1993). Éléments d'une modélisation de l'acte d'enseigner : planification et décision. Communication au premier Congrès d'actualité de la recherche en éducation et formation. Paris : APRIEF.

(23) CADET, B. (1990). Déterminants décisionnels des actions pédagogiques. In J. ARDOINO, G. MIALARET (Eds), Les nouvelles formes de la recherche en éducation. Paris : Matrice-ANDSHA, 261269 ; SHAVELSON, R. J., CADWELL, J., IZU, T. (1977). Teachers' Sensitivity to the reliability of Information in making Pedagogical Decisions. American Educational Research Journal, 14-2, 83-97.

(24) Voir RIFF, J., DURAND, M. (1993). Planification et décision chez les enseignants. Revue française de pédagogie, 103, 81-107. 


\section{DISCUSSION, OU COMPRENDRE POUR TRANSFORMER L'ACTIVITÉ DE L'ENSEIGNANT}

Nous ne prétendons pas avoir développé toutes les images que les chercheurs ont pu attribuer à l'enseignant. Plus récentes, des théories d'inspiration psychanalytique (25), vygotskienne (26) ou socioconstructiviste (27) enrichissent l'approche que l'on peut avoir du phénomène de l'enseignement. Heureusement ou malheureusement, il n'est même pas certain que les images recensées ci-dessus recouvrent la totalité des pratiques enseignantes, voire ne se recoupent pas entre elles.

Le problème actuel n'est pas de faire cohabiter ces différentes et nombreuses acceptions de l'activité de l'enseignant ; il serait plutôt, à l'inverse, d'empêcher qu'elles se mélangent. Ainsi, Bourdoncle (28) montre, à propos de l'utilisation des modèles de formation professionnelle, qu'un trop grand éclectisme dans leur provenance — fonctionnaliste, interactionniste ou critique — peut nuire à leur clarté.

Pour éviter que les images à propos des enseignants ne deviennent des clichés de plus en plus flous, il est nécessaire de leur appliquer de sévères critères de sélection. Ainsi, les images à visée descriptive pourront être soumises à expérimentation, ce qui n'est pas toujours le cas, notamment pour celles concernant l'enseignant réfléchi. De même, les images à visée prescriptive pourront être liées à une efficacité dans le domaine de la formation des enseignants ce qui, là encore, peut être vérifié expérimentalement.

Pour terminer, nous voudrions souligner l'intérêt qu'il y aurait de procéder en coordonnant les approches descriptives et prescriptives. On pourrait pour cela adopter une démarche ergonomique (29) : comprendre l'activité de l'enseignant (la décrire) pour mieux la transformer (prescrire des aides adéquates). Cette attitude éviterait la démarche tautologique souvent employée, notamment en formation d'enseignants : prescrire une méthode d'enseignement, de planification, pour ensuite la décrire et s'en

(25) Notamment WAGNER, A. C. (1987). "Knots" in teachers' thinking. In J. CALDERHEAD (Ed.), Exploring teachers' thinking. Londres : Cassel, 161-178; qui modélise la gestion rationnelle des problèmes cognitifs et affectifs (stress, anxiété, attachement...) pouvant se poser à l'enseignant.

(26) Voir MANNING, B. H., PAYNE, B. D. (1993). A vygotskian-based theory of teacher cognition. Teacher and Teaching Education, 9-4, 361-371, qui testent l'hypothèse d'une autorégulation des registres cognitifs et affectifs de l'enseignant.

(27) Voir CRAHAY, M. (En préparation). Théories implicites de l'éducation et pensée réflexive des enseignants : un recadrage socio-constructiviste. Université de Liège, manuscrit non publié. Crahay reconsidère le paradigme de l'enseignant réfléchi à la lumière des théories piagétiennes. L'enseignant, par un jeu d'accomodations/assimilations, pourrait se défaire de ses théories implicites de l'éducation afin de repenser son action et d'enrichir sa pratique.

(28) Op. cit.

(29) Nous reprenons ici e titre de GUÉRIN, F., LAVILLE, A., DANIELLOU, F., DURAFFOURG, J., KERGUELEN, A. (1991). Comprendre le travail pour le transformer, la pratique de l'ergonomie. Montrouge : ANACT, coll. Outils et méthodes. 
déclarer satisfait, en oubliant qu'elle peut ne pas répondre aux besoins des principaux intéressés.

Nous voudrions enfin éclairer une image qui, bien qu'encore marginale, nous semble porteuse de nouveaux travaux : celle de l'enseignant «bricoleur ». Reprise de Lévi-Strauss (30), elle développe l'idée que l'enseignement s'apparente à un art(isanat) plutôt qu'à une science et que l'activité de l'enseignant est un assemblage constamment en déséquilibre. Pour Perrenoud, l'enseignant ferait «avec les moyens du bord », en assemblant, désassemblant, polissant chacune de ses préparations afin qu'elle corresponde au mieux à ses élèves :

« bricoler, c'est résoudre un problème avec des matériaux partiellement inadéquats. [... l'enseignant] est sans cesse en train de combiner et d'adopter, voire de créer des moyens d'enseignement, des situations didactiques convenant à ses élèves et à la façon dont il progresse dans son programme » (31).

La gêne des chercheurs à trouver un paradigme commun pour l'étude de l'enseignant participe de la difficulté qu'ont les Sciences de l'éducation à former un groupe de savoirs formalisés. Une unité aurait pu être trouvée dans le champ de recherche sur la pensée des enseignants. Prometteur jusqu'à la fin des années quatre-vingt (32), il semble se tarir (33), pour de multiples raisons, dont la principale est sans doute qu'en restant exclusivement descriptif, il n'a pas alimenté les modèles de formation des enseignants, à visée plus pragmatique. Les recherches à venir, pour ne pas rester lettre morte, devront donc tenir compte de ce difficile passage du descriptif au prescriptif.

(30) LÉVI-STRAUSS, C. (1962). La pensée sauvage. Paris : Plon ; repris par PERRENOUD, P. (1983). La pratique pédagogique entre l'improvisation réglée et le bricolage. Éducation et recherche, 2, 198-212 ; TOCHON, F. V. (1992). A quoi pensent les chercheurs quand ils pensent aux enseignants? Revue Française de Pédagogie, 99, 89-113.

(31) PERRENOUD (1983, op. cit.).

(32) Voir, pour une synthèse, TOCHON, F. V. (1989). «La pensée des enseignants » : un paradigme en développement. Perspectives documentaires en éducation, 17, 75-97.

(33) Nous parlons bien évidemment des recherches anglosaxonnes, car les recherches francophones n'ont jamais été très nombreuses. Voir toutefois CHARLIER, E. (1989). Planifier un cours, c'est prendre des décisions. Bruxelles : De Boeck ; CRAHAY, M. (1989). Contraintes de situation et interactions maître-élève, changer sa façon d'enseigner, est-ce possible ? Revue Française de Pédagogie, 88, 67-94 ; et RIFF, J., DURAND, M., op. cit. 